\title{
Smoking among young urban Malaysian women and its risk factors.
}

\begin{abstract}
This study was conducted to measure the prevalence of cigarette smoking and to determine the individual, family, and environmental factors associated with smoking among young urban women. A cross-sectional study through self-administered questionnaire was conducted on female students enrolled in private higher learning institutions in Kuala Lumpur and Selangor, Malaysia, between July and October 2005. Analysis on 408 respondents showed that current smoker prevalence rate was 18.6\%. Adjusted analyses showed significant association between smoking and individual factors, which are the importance of slim image, average monthly allowance, and car ownership. For family factors, analyses showed significant association between smoking and parental marital status and smoking status of male siblings. Strong associations were seen between female smoking and environmental factors, such as having more smoker friends, having smokers as best friends, keeping cigarette-brand items, being offered free cigarette, and perceiving female smoking as normal. The identified risk factors could be used to develop more effective prevention programs to overcome smoking among young urban women.
\end{abstract}

Keyword: Malaysia; Prevalence; Risk factors; Smoking; Urban; Young women 